\section{Exacerbation of diazepam-induced phlebitis by oral penicillamine}

Penicillamine, a heavy metal antagonist, is used in the treatment of Wilson's disease, cystinuria, rheumatoid arthritis, and various other conditions. Adverse reactions to the drug are many and frequent and range in severity from gastrointestinal distress, stomatitis, unpleasant skin odours, and rashes to proteinuria, leucopenia, and thrombocytopenia. ${ }^{1} \mathrm{Up}$ to one-third of patients given the drug for rheumatoid arthritis have to discontinue treatment because of toxicity. ${ }^{1}$ Intravenous diazepam is widely used as a sedative, muscle relaxant, and anticonvulsant. Local complications with this drug, including pain at injection site, phlebitis, and thrombophlebitis, have likewise been reported frequently."

We describe a patient who developed phlebitis from intravenous diazepam. The phlebitis resolved with local heat but recurred on two separate occasions after oral penicillamine.

\section{Case report}

A 33-year-old white man underwent endoscopic retrograde cholepancreatography for investigation of persistent raised activity of alkaline phosphatase and 5'-nucleotidase. He had had ulcerative colitis 10 years earlier which had been effectively treated with sulphasalazine. Five years later an asymptomatic rise of alkaline phosphatase and $5^{\prime}$-nucleotidase activities and findings on oral cholecystogram consistent with cholecystitis prompted a cholecystectomy. This showed acalculus cholecystitis. Minimal biliary duct inflammation was found on liver biopsy. Although the patient had remained asymptomatic the alkaline phosphatase activity remained raised at 70 to $225 \mathrm{IU} / 1$ (normal 13-40) as did the 5 '-nucleotidase activity at more than 17 Bodansky units (normal 0.3-3.2). Results of tests for antimitochondrial and antismooth muscle antibodies were negative and serum bile acid concentrations were normal. An endoscopic retrograde cholepancreatography was performed using $5 \mathrm{mg}$ of diazepam intravenously for sedation. This procedure showed mild beading and narrowing of the biliary ducts consistent with sclerosing cholangitis. Two days later mild phlebitis developed in the right forearm in the region of the antecubital fossa where diazepam had been injected. The pain, erythema, and warmth resolved after one week of treatment with local heat. Two weeks later penicillamine, in a daily dose of $250 \mathrm{mg}$ orally, was started. Four days later the phlebitis recurred and persisted for the next two weeks. Penicillamine was discontinued, and the phlebitis resolved over three weeks in response to local heat. As haematological and renal values were stable and there were no other side effects penicillamine was restarted at the same dose. Two days later phlebitis recurred. Penicillamine was immediately discontinued, and the phlebitis resolved over four weeks. The patient was re-evaluated by another doctor, who felt that the clinical history and results on endoscopic retrograde cholepancreatography were more consistant with pericholangitis, and prednisone treatment was started. The alkaline phosphatase and $5^{\prime}$-nucleotidase activities returned to normal after five months of treatment, and prednisone was tapered off without adverse effects.

\section{Comment}

Phlebitis is a local inflammatory condition of the vein without induration characterised by erythema, oedema, and pain. Local complications after diazepam commonly start 24 to 48 hours after intravenous administration and last from a few days to several weeks." Local treatment with heat is generally adequate, though antibiotics, anticoagulants, analgesics, or anti-inflammatory agents are sometimes used. Diazepam-induced local complications appear to be caused by an inflammatory reaction directly related to the chemical irritation of diazepam or its vehicle. ${ }^{2}$

The treatment of sclerosing cholangitis, a condition that may result in biliary cirrhosis and death, is not satisfactory. Since penicillamine has been used experimentally for primary biliary cirrhosis with encouraging results ${ }^{3}$ its use in sclerosing cholangitis seemed reasonable as a therapeutic trial.

The finding that phlebitis, presumably from intravenous diazepam, recurred after apparent healing after two separate courses of oral penicillamine was interesting. We found only one report describing the development of thrombophlebitis after beginning penicillamine treatment in a patient with a history of recurrent phlebitis. ${ }^{4}$ The thrombophlebitis resolved shortly after discontinuation of penicillamine.

Penicillamine has an influence on collagen synthesis which appears to be dose-related. Evidence in animals treated with penicillamine suggests that wound healing is defective, possibly due to production of poor quality collagen. ${ }^{5}$ In this case the phlebitis though clinically resolved may still have been healing. Penicillamine may have prevented this subclinical healing and caused the exacerbation of phlebitis.

${ }^{1}$ Kean WF, Dwosh IL, Anastassiades TP, Ford PM, Kelly HG. The toxicity pattern of D-penicillamine therapy-guide to its use in rheumatoid arthritis. Arthritis Rheum $1980 ; 23: 158-64$.

2 Donaldson D, Gibson G. Local complications with intravenous diazepam. Canadian Dental Association fournal 1979;45:337-41.

${ }^{3}$ Dickson ER, Fleming CR, Ludwig J. Primary biliary cirrhosis. Prog Liver Dis $1979 ; 6: 487-502$

${ }^{4}$ Davis CM. D-penicillamine for the treatment of gold dermatitis. $\mathrm{Am} \mathcal{F}$ Med $1969 ; 46: 472-4$

${ }^{5}$ Burry HC. Penicillamine and wound healing-a potential hazard? Postgrad Med 7 1974;50, suppl 2:75-6.

(Accepted 26 May 1981)

Pulmonary Unit, Massachusetts General Hospital, Boston MA 02114 ROBERT D BRANDSTETTER, MD, fellow

Department of Pharmacy Practice, College of Pharmacy, University of Florida, Gainesville, FL 32601

VINCENT P GOTZ, MS, assistant professor

Pharmacy Division, New York Hospital, New York

DEXTER D MAR, PHARMD, pharmacist

Dietary Division, Massachusetts General Hospital, Boston MA 02114

DEANE SACHS, BS, dietitian

\section{Reversible renal damage due to glue sniffing}

Glue sniffing and other forms of solvent abuse are becoming increasingly common. Toxic effects are rarely encountered, and a recent survey showed no evidence of renal or hepatic damage in 132 cases. $^{1}$ We report a case of reversible renal damage caused by sniffing a proprietary adhesive-one of the most widely abused agents in Britain-whose main solvents are toluene and acetone.

\section{Case report}

A 14 -year-old boy presented to his general practitioner with a 24 -hour history of sore throat and was prescribed cephalexin. The next day he returned complaining of haematuria, urinary frequency, and bilateral loin pain and was referred to hospital. He had abused solvents for two years and for eight months had "sniffed" a pint of adhesive daily. After three weeks of abstinence he had sniffed one pint of adhesive on each of the three days before his admission to hospital.

On examination he was flushed and had a fever of $38 \cdot 6 \mathrm{C}$. Scabbed excoriations were present on the lower lip. The fauces were red and inflamed, his face was puffy, and several cervical nodes were palpable. There was no evidence of peripheraloedema or raised jugular venous pulsation. Blood pressure was $140 / 80 \mathrm{~mm} \mathrm{Hg}$ on admission; this was the highest level recorded during his stay. The only other abnormal finding was bilateral loin tenderness. Plasma urea and creatinine concentrations on admission were normal. Urine analysis showed blood $t+t$ and protein $t+t$, and microscopy showed red blood cells but no casts.

In the 24 hours after admission he passed only $200 \mathrm{ml}$ of urine, his face became more swollen, and the plasma urea concentration rose to $10.3 \mathrm{mmol} / 1$ $(61.9 \mathrm{mg} / 100 \mathrm{ml})$ and creatinine concentration to $132 \mathrm{mmol} / 1 / 1493 \mathrm{mg} / 100$ $\mathrm{ml}$ ). Urine contained $2 \cdot 2 \mathrm{~g}$ protein $/ \mathrm{l}$. Liver function tests showed normal results. Blood cultures, three midstream specimens of urine, and two throat swabs showed no growth. Antinuclear factor and antistreptolysin 0 titre were negative. Fluid intake was restricted and renal function closely monitored. Urine output increased daily, and by the fourth day after admission he was no longer oliguric. Plasma urea and creatinine concentrations returned to normal on the fifth day. Four weeks later his urine was free of blood, and protein and plasma urea and creatinine concentrations remained normal. Creatinine clearance was $112 \mathrm{ml} / \mathrm{min}$ 